\title{
Experimental Measurements and Multi-Scale Modeling of the Relative Gas Permeability of a Caprock
}

\author{
François Bignonnet ${ }^{1,2,3, *}$, Zhibo Duan ${ }^{2}$, Patrick Egermann ${ }^{4}$, Laurent Jeannin ${ }^{5}$ \\ and Frédéric Skoczylas ${ }^{2}$ \\ ${ }^{1}$ LUNAM Université, GeM, UMR CNRS 6183, École Centrale Nantes, Université de Nantes, 44600 Saint-Nazaire - France \\ 2 École Centrale de Lille, Laboratoire de Mécanique de Lille (UMR 8107), 59655 Villeneuve d'Ascq - France \\ 3 Université Paris-Est, Laboratoire Navier (UMR 8205), CNRS, ENPC, IFSTTAR, 77455 Marne-la-Vallée - France \\ ${ }^{4}$ Storengy, Immeuble Diinn, 12 rue Raoul Nordling, 92270 Bois-Colombes - France \\ ${ }^{5}$ ENGIE E\&P International, Faubourg de l'Arche, 1 place Samuel de Champlain, 92930 La Défense - France \\ e-mail: francois.bignonnet@univ-nantes.fr \\ * Corresponding author
}

\begin{abstract}
An experimental characterisation of a low permeability caprock is first reported. Two different methods are compared to measure the porosity: water imbibition and weighting versus gas (Argon) pycnometry. Water measured porosities appear to systematically underestimate gas measured porosities. A capillary pressure curve is derived from successive equilibrium of samples at imposed relative humidities. The relative gas permeability is thoroughly measured on the same samples. Gas porosity and effective gas permeability measurements have been carried out under different confining pressures. All measured relative gas permeabilities lie on a master curve and present a low critical water saturation beyond which the effective gas permeability nearly vanishes. In an attempt to model this last feature, the salient properties of the microstructure are extracted from electron microscopy images to propose a micro-macro model of the relative gas permeability. The multi-scale modeling is performed within the framework of random media or so-called continuum micromechanics, including localised flow effects within interfaces. The self-consistent homogenisation scheme allows retrieval of a critical water saturation, whose value is governed by the shape of the elementary particles of the material and the localisation of water within the pore space.
\end{abstract}

Résumé - Caractérisation expérimentale et modélisation multi-échelles de la perméabilité elative au gaz d'une roche de couverture - Une caractérisation expérimentale d'une roche carbonatée contenant de l'argile, peu perméable, est présentée dans un premier temps. Deux méthodes différentes de mesure de la porosité sont comparées : gravitaire par imbibition d'eau et pycnométrie à l'Argon. Les mesures de porosité à l'eau sous-estiment systématiquement celles au gaz. Une courbe de pression capillaire est construite à partir d'équilibres successifs d'échantillons avec des humidités relatives imposées. La perméabilité relative au gaz est mesurée sur les mêmes échantillons. Les mesures de perméabilité effective au gaz et de porosité au gaz sont conduites sous diverses pressions de confinement. Toutes les perméabilités relatives au gaz mesurées appartiennent à une même courbe maîtresse. Cette courbe présente une faible saturation en eau critique au delà de laquelle la perméabilité effective au gaz s'annule. Dans un second temps, une tentative de modélisation de cette propriété est proposée. Un modèle micro-macro est proposé à partir d'observation de la microstructure au microscope électronique. La modélisation multi-échelles est conduite dans le contexte de l'homogénéisation des milieux aléatoires ou micromécanique des 
milieux continus et prend en compte une localisation de l'écoulement dans des interfaces entre constituants. L'utilisation du schéma auto-cohérent permet d'exhiber une saturation en eau critique, dont la valeur dépend de la forme des particules élémentaires ainsi que de la répartition de l'eau au sein de l'espace poreux.

\section{INTRODUCTION}

Low permeability caprock characterisation is important for both subsurface storage activities (Carbon Capture \& Sequestration, gas, etc.) and unconventional resource production. In most published papers, emphasis is put on the threshold capillary pressure characterisation. However, the available literature data about the associated relative permeabilities is scarce although it also contributes to either the confinement of storage facilities or the production rates of wells. This is mainly due to the complexity of deriving these data in a representative manner from laboratory experiments in a reasonable time frame. This work is a contribution to provide such data from a tight carbonate rock with clay content and to propose an approach to interpret their shapes.

Relative gas permeability measurements can be performed in a reasonable time frame on standard permeable media, such as conventional sandstones [1-3]. However, relative gas permeability measurements on weakly permeable media remain challenging. Recent developments have allowed the study of low permeability materials, such as concrete [4] and tight sandstones [5].

The present study focuses on a tight carbonate rock with clay content, extracted from a caprock facies interval. First, the relative gas permeability is assessed experimentally with respect to partial water saturation. To get a deeper understanding of the porous network properties, two porosity measurement methods are used and a capillary pressure curve is determined (Sect. 1 and 2). Second, an analytical permeability upscaling model is proposed and compared to experimental data in Section 3.

\section{MATERIAL AND METHODS}

\subsection{Material}

\subsubsection{Sample Origin and Macroscopic Description}

The studied material comes from a single core extracted at a depth of $450 \mathrm{~m}$. In the study, a confining pressure of $9 \mathrm{MPa}$ has been assumed to be representative of the in-situ hydrostatic pressure. At the macroscopic scale, the material is of the type "mudrock", very fine grained, mostly carbonated and fairly homogeneous (Fig. 1). Geochemical analysis indicates that the carbonate content is high with 276,000 parts per million in weight (ppm) of $\mathrm{Ca}$, located in the light-gray regions. It also features a minor component of pyrite $(1.1 \%)$ as well as silicates and clay as deduced from the measured values of 7,030 ppm of $\mathrm{Mg}, 7,980 \mathrm{ppm}$ of $\mathrm{K}, 11,250 \mathrm{ppm}$ of Fe and 6,130 ppm of S. EDX analysis indicates that the clay content is located in the dark-grey regions and is most likely illite. The total organic carbon is $0.57 \%$.

\subsubsection{Microscopic Description}

A petrographic analysis using Scanning Electron Microscopy (SEM) shows at a low magnitude of amplification (Fig. 2a) a microstructure of the type "grain-supported", that is with carbonated grains (light gray) in contact. The size of the carbonated grains ranges from 1 to $10 \mu \mathrm{m}$ approximately. Pyrite spheres (in white) and silicate silts (dark grey) smaller than $10 \mu \mathrm{m}$ are embedded in this matrix. Observation at a finer scale (Fig. 2b) shows that the carbonates (light grey) tend to form clusters or aggregates with low porosity. The porosity is rather located in micro-metric regions with a high concentration of clay particles (dark grey) and inter-particle porosity (black). These high porosity regions are seemingly connected through thin "canals" crossing the carbonate aggregates.

\subsubsection{Sample Preparation}

Eight vertical cylindrical samples with diameter $37 \mathrm{~mm}$ and height $10 \mathrm{~mm}$ have been prepared from the main vertical core, so that the axis of the cylinders is assumed perpendicular to the bedding. First, longer cylinders are obtained by coring. Second, the long cylinders are cut into several $10 \mathrm{~mm}$ height cylinders with a wire saw. The upper and lower faces of the samples are then polished with sandpaper. The height $h$ and diameter $d$ of each sample are then measured with a calliper with a precision of $0.02 \mathrm{~mm}$. The total volume $V$ of each sample is simply assessed by $V=\pi h d^{2} / 4$.

\subsection{Methods}

\subsubsection{Partial Water Saturation by Relative Humidity Control}

All samples are first oven-dried at $60^{\circ} \mathrm{C}$ until mass stabilisation is achieved, which occurs after about two weeks. This state is considered as the reference dry state, and the water saturation $S_{w}$ is assumed null. The mass in the dry state $m_{\mathrm{dry}}$ is measured with a precision of $0.01 \mathrm{~g}$. 


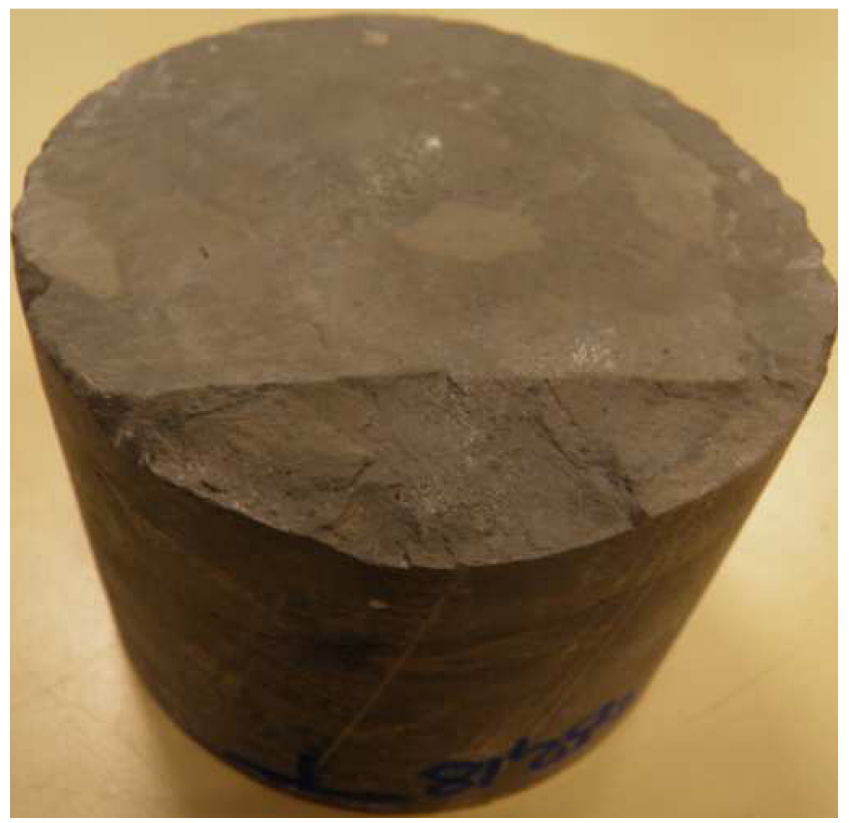

Figure 1

Macroscopic aspect (core diameter is $\approx 10 \mathrm{~cm}$ ).

To obtain a partial water saturation, the samples are stored in a chamber comprising air and water vapour in equilibrium with a saturated saline solution at $20^{\circ} \mathrm{C}$ [4]. The relative humidity of the atmosphere in the chamber is imposed by thermo-dynamical equilibrium and depends on the type of saline solution. The relative humidities achieved by this method are reported in Table 1.

Since the chemical activities of liquid water and vapour at equilibrium are equal, the capillary pressure $P_{\text {cap }}=P_{g}-P_{l}$ between the gas (here at atmospheric pressure) and the liquid water (which condenses in the pore network of the sample) is related to the relative humidity $r h$ of the atmosphere by Kelvin's law:

$$
P_{\text {cap }}=-\frac{\rho_{l} R T}{M} \ln (r h)
$$

where $\rho_{l}$ is the density of water, $M$ the molar mass of the water, $R$ the perfect gas constant and $T$ the temperature. For water at $20^{\circ} \mathrm{C}$, the capillary pressures corresponding to the imposed relative humidities are reported in Table 1.

Moreover, the capillary pressure is related to the curvature $C$ of the liquid-gas meniscii by Young-Laplace's law:

$$
P_{\text {cap }}=\gamma C
$$

where $\gamma$ is the coefficient of surface tension, equal to $0.073 \mathrm{~N} . \mathrm{m}^{-1}$ under ambient conditions. If the contact angle

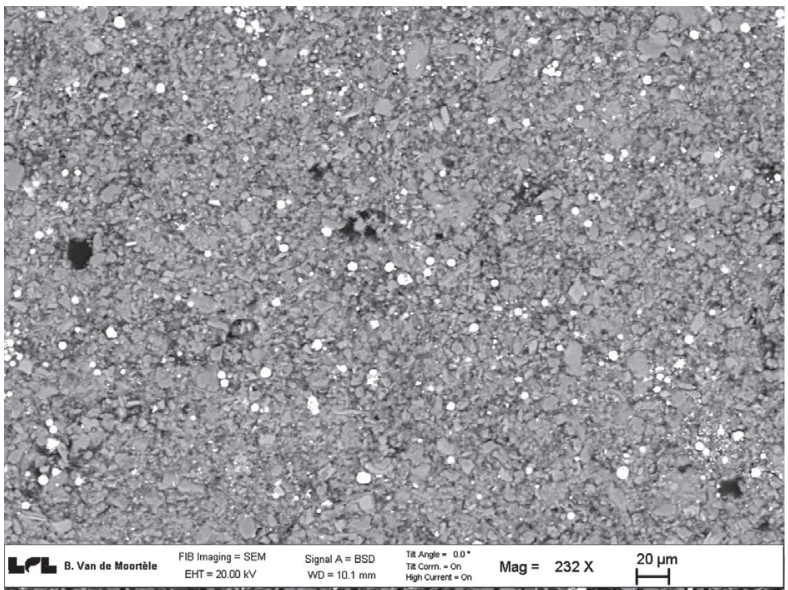

a)

Low magnitude SEM observation

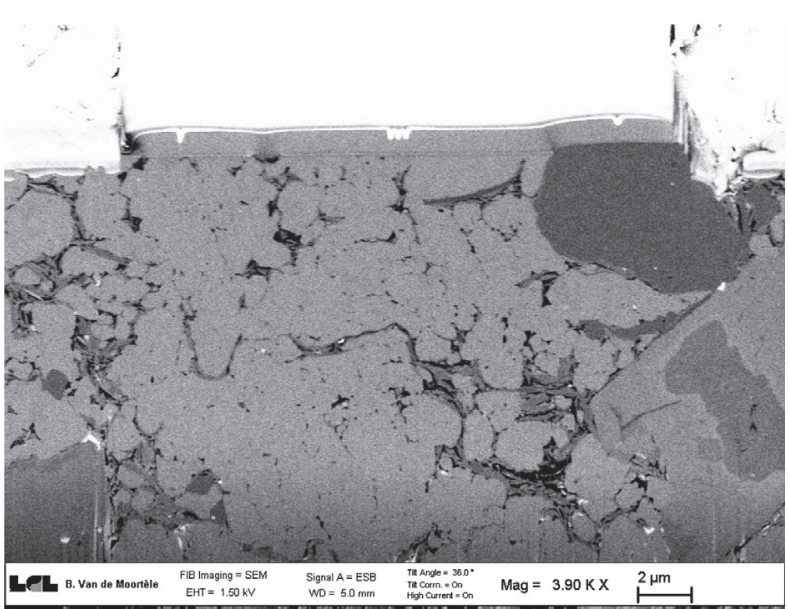

b) FIB abrasion and high magnitude SEM observation

Figure 2

SEM observations of the microstructure by Bertrand van de Moortèle (ENS Lyon).

between the water and the solid phase is null, the curvature of the liquid-gas interface in a cylinder of radius $r$ is $C=2 / r$. In the case of a meniscus in-between two planes spaced by an aperture $e$ (the case of a "flat pore"), the curvature is $C=2 / e$. For water at $20^{\circ} \mathrm{C}$, the cylinder radii and flat pore apertures corresponding to the imposed relative humidities through the Kelvin-Laplace law are reported in Table 1.

A sample successively equilibrated in chambers with increasing relative humidity undergoes a progressive imbibition, from the smaller to the larger pores. At each step, equilibrium is assumed once mass stabilisation of the sample is achieved, which occurs after about two weeks. The mass 
TABLE 1

Imposed relative humidities $(r h)$ and corresponding capillary pressures $\left(P_{\text {cap }}\right)$ and cylinder radii $(r)$ or flat-pore aperture $(e)$ using Equations (1) and (2)

\begin{tabular}{c|c|c}
\hline$r h(\%)$ & $P_{\text {cap }}(\mathrm{MPa})$ & $r$ or $e(\mathrm{~nm})$ \\
\hline 11 & 298.6 & 0.49 \\
\hline 43 & 1142 & 1.27 \\
\hline 59 & 71.39 & 2.04 \\
\hline 73 & 42.58 & 3.42 \\
\hline 85 & 21.99 & 6.62 \\
\hline 92 & 11.28 & 12.9 \\
\hline 98 & 2.733 & 53.3 \\
\hline 100 & 0 & - \\
\hline
\end{tabular}

$m(r h)$ in the partially water saturated state due to the relative humidity $r h$ is measured with a precision of $0.01 \mathrm{~g}$. Note that the water saturation is not directly known at this stage.

At the end of the progressive imbibition process by successive equilibrium in chambers with increasing relative humidities up to $100 \%$, the samples are immersed in water (partially immersed for the 6 first hours to allow the air to drain, then fully immersed) in a vacuumed chamber for 24 hours. The mass in this saturated state $m_{\text {sat }}$ is measured.

Assuming the final immersion step guarantees a total water saturation of the sample, a way to determine the porosity $\phi$ is:

$$
\phi=\frac{m_{\mathrm{sat}}-m_{\mathrm{dry}}}{V_{\rho_{l}}}
$$

The relation (3) provides a preliminary way of measuring the porosity. Porosity measured using this method will be referred to as "water porosity". This method will be compared to another method later in the paper.

Additionally, the partial water saturation $S_{w}(r h)$ achieved at any of the previous steps by equilibrium with relative humidity $r h$ may now be assessed using:

$$
S_{w}(r h)=\frac{m(r h)-m_{\mathrm{dry}}}{m_{\mathrm{sat}}-m_{\mathrm{dry}}}
$$

The $S_{w}-r h$ curve obtained from Equation (4) can be used to derive the capillary pressure curve by means of Kelvin's law (1). The latter may be used with caution to estimate the pore size distribution through Young-Laplace's law (2), provided strong assumptions on the shape (cylindrical or flat pores for example) and connectivity of the pores are made.

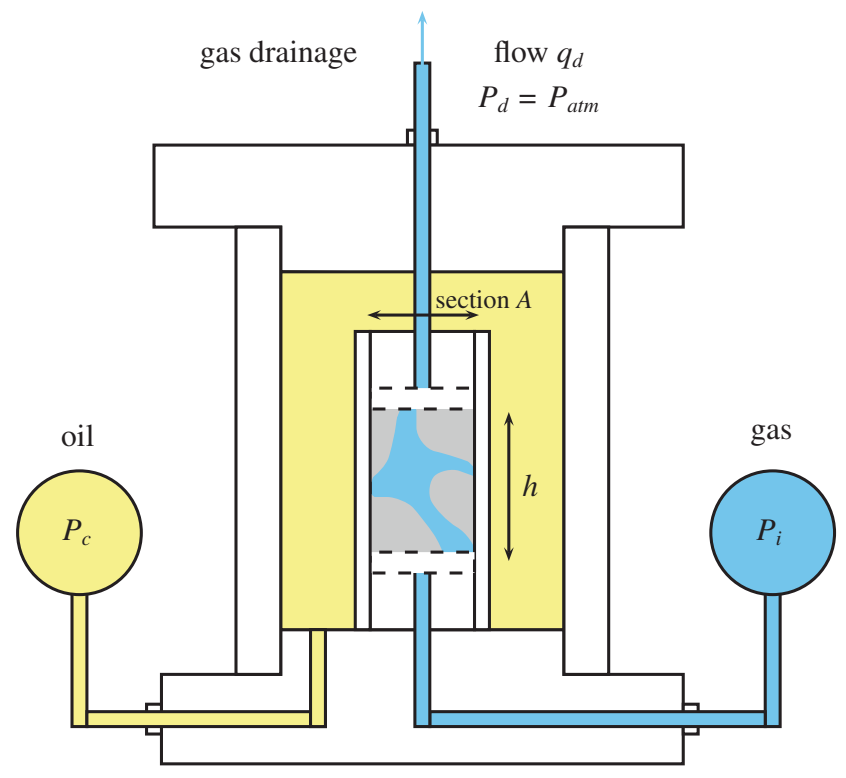

Figure 3

Uniaxial steady state gas permeameter with confining pressure $P_{c}$, gas injection and drainage pressures $P_{i}$ and $P_{d}$ respectively, and sample height $h . P_{\text {atm }}$ is the atmospheric pressure.

\subsubsection{Gas Permeability Measurements}

\section{Steady State Apparatus}

Gas permeability $K$ is measured using a uniaxial steady state gas flow apparatus (Fig. 3). The apparatus consists of a confining cell which is allowed to reach confining pressures $P_{c}$ as high as $60 \mathrm{MPa}$, together with a gas injection device [1, 4-9]. The gas used in the current study is 99\%-pure Argon with a viscosity of $\mu=2.2 \times 10^{-5}$ Pa.s.

Let $x=0$ be the coordinate of the injection (or upstream) side of the sample and $x=h$ the drainage (or downstream) side. The injection pressure $P_{i}$ at $x=0$ is set to values ranging from $0.5 \mathrm{MPa}$ to $1.5 \mathrm{MPa}$, whereas the back-pressure $P_{d}$ at $x=h$ is equal to the atmospheric pressure. The injection pressure is measured with a pressure sensor with a precision of $0.002 \mathrm{MPa}$. The drainage flow rate $q_{d}$ at $x=h$ is measured with a set of flow-meters, the finer of them allowing measurement of flow rates from $0.1 \mathrm{~mL} / \mathrm{min}$ to $1 \mathrm{~mL} / \mathrm{min}$ with a precision of $0.01 \mathrm{~mL} / \mathrm{min}$.

\section{No-Slip Flow}

In the case where gas-slippage effects can be neglected, combining Darcy's law and the perfect gas law to mass 
conservation yields the uniaxial diffusivity equation describing the pressure $p(x, t)$ in the sample:

$$
\frac{\partial}{\partial t}(\phi p(x, t))=\frac{\partial}{\partial x}\left(p(x, t) \frac{K}{\mu} \frac{\partial p(x, t)}{\partial x}\right)
$$

In a steady state test, boundary conditions constrain the pressure profile inside the sample to be:

$$
p(x)=\sqrt{P_{i}^{2}-\left(P_{i}^{2}-P_{d}^{2}\right) \frac{x}{h}} \quad(0 \leq x \leq h)
$$

so that the pressure gradient is $\left(P_{d}^{2}-P_{i}^{2}\right) /(2 p(x) h)$. Combining Darcy's law and flow continuity at $x=h$ yields the expression for the permeability $K$ as a function of the measured drainage flow rate $q_{d}$ :

$$
K=\frac{2 \mu h P_{d} q_{d}}{A\left(P_{i}^{2}-P_{d}^{2}\right)}
$$

where $A=\pi d^{2} / 4$ is the area of the sample section.

\section{Slip Flow}

In the case where the local permeability is governed by Klinkenberg's slippage law [10], the apparent local permeability depends on the gas pressure as:

$$
K^{\mathrm{app}}(p(x))=K^{\mathrm{int}}\left(1+\frac{\beta}{p(x)}\right)
$$

where $K^{\text {int }}$ is the intrinsic gas permeability and $\beta$ is Klinkenberg's coefficient. Carefully introducing (8) into (5) and considerations similar to the no-slip case rigorously leads to:

$$
K_{m}^{\mathrm{app}}=K^{\mathrm{int}}\left(1+\frac{\beta}{P_{m}}\right)=\frac{2 \mu h P_{d} q_{d}}{A\left(P_{i}^{2}-P_{d}^{2}\right)} \quad \text { with } \quad P_{m}=\frac{P_{i}+P_{d}}{2}
$$

where $P_{m}$ is a mean pressure and $K_{m}^{\text {app }}$ is a mean apparent permeability, in an averaged way along the sample. The similarity of the relations (9) with (8) and (7) is striking and convenient, but was non-obvious a priori and is merely due to the particular form of the pressure dependence of the permeability in Klinkenberg's law. Hence, the plot of $K_{m}^{\text {app }}$ with respect to $1 / P_{m}$ allows determination of the intrinsic gas permeability and the Klinkenberg coefficient, irrespective of the fact that $P_{i}$ and $P_{d}$ may be very different. In practice, three different gas injection pressures are successively applied: $0.5,1$ and $1.5 \mathrm{MPa}$.

\section{Effective Gas Permeability}

In order to assess the relative gas permeability as a function of the water saturation $S_{w}$, effective gas permeability

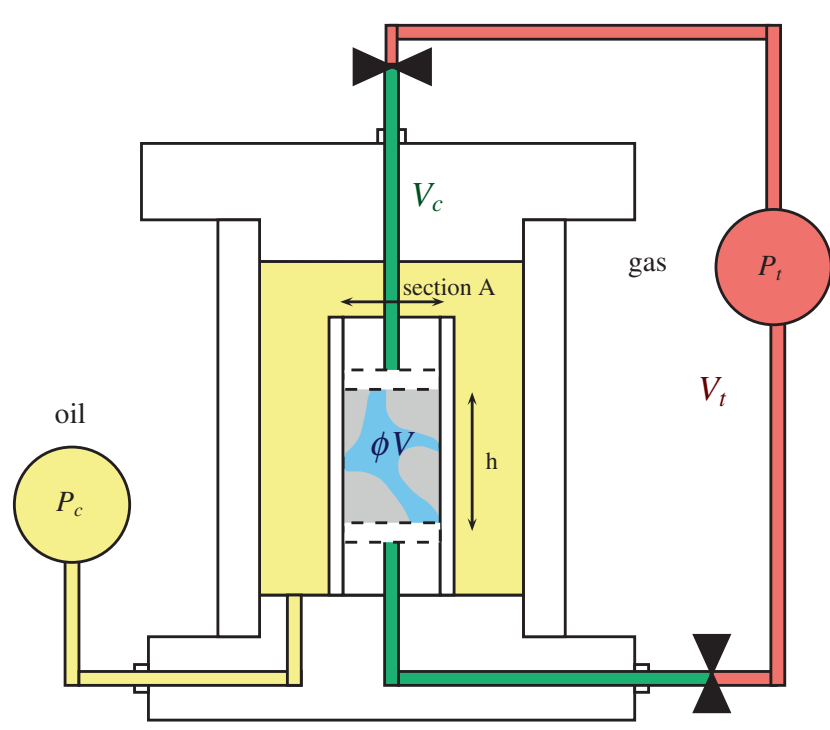

Figure 4

Gas porosity measurement apparatus with confining pressure $P_{c}$, initial reference tank pressure $P_{t}$. The reference tank has a calibrated volume $V_{t}$, the connecting tubes' volume is $V_{c}$. The total volume of the sample is $V$ and its porosity is $\phi$.

measurements are performed at each step of saturation obtained by the procedure described in Section 1.2.1. As the Argon gas injected in the partially water saturated sample is dry, the mass of the sample is checked before and after the measurement to monitor potential desaturation of the sample. In practice for the current study, changes in water saturation due to dry gas injection have been observed to be negligible.

\subsubsection{Gas Porosity Measurements}

The porosity has been measured at three steps of loading/ unloading of the dry gas permeability measurements using a dedicated method [6], similar in principle to gas pycnometry.

The measurement is performed in the same confining cell as for the permeability test (Fig. 4). The room temperature is set to $20^{\circ} \mathrm{C}$. Three volumes have to be distinguished:

- a reference tank with a calibrated volume $V_{t}$ of $70 \mathrm{~mL}$,

- the pore volume $\phi \times V$ to be measured,

- the volume $V_{c}$ of the tubes connecting the reference tank to both sides of the sample.

The reference tank is initially at a pressure $P_{t}$ of $1 \mathrm{MPa}$ which is monitored using a pressure sensor with a precision of $100 \mathrm{~Pa}$, whereas the porosity and the connecting tubes are at atmospheric pressure $P_{\text {atm }}$. The valves between the reference tank and the sample are then opened while the pressure in the reference tank is monitored. Once gas pressure and 
TABLE 2

Measured water porosities and gas porosities

\begin{tabular}{|c|c|c|c|c|}
\hline & \multicolumn{3}{|c|}{ Gas (Argon) } & Water \\
\hline & \multicolumn{4}{|c|}{ Confining pressure $(\mathrm{MPa})$} \\
\hline \multirow[b]{2}{*}{ Sample } & 2 & 9 & 2 & 0 \\
\hline & \multicolumn{4}{|c|}{ Porosity $\phi(\%)$} \\
\hline MIL-12 & - & - & - & 10.6 \\
\hline MIL-13 & 11.84 & 10.67 & 11.53 & 9.6 \\
\hline MIL-14 & 12.43 & 11.69 & 12.15 & 8.9 \\
\hline MIL-20 & - & - & - & 14.5 \\
\hline MIL-21 & - & - & - & 13.3 \\
\hline MIL-22 & 12.70 & 11.79 & 12.50 & 10.2 \\
\hline MIL-23 & 11.65 & 10.81 & 11.51 & 9.0 \\
\hline MIL-24 & 11.75 & 11.13 & 11.77 & 9.1 \\
\hline
\end{tabular}

temperature are equilibrated, application of the law of perfect gases in isothermal conditions links the final pressure $P_{f}$ to the sample porosity through:

$$
P_{t} V_{t}+P_{\mathrm{atm}}\left(V_{c}+\phi V\right)=P_{f}\left(V_{t}+V_{c}+\phi V\right)
$$

The measure of porosity deduced from Equation (10) is referred to as "gas porosity". The volume $V_{c}(\approx 5 \mathrm{~mL})$ is measured based on the same principle using a non-porous steel sample.

\section{EXPERIMENTAL RESULTS}

\subsection{Porosity}

The measured "water porosities" (Eq. 3) and "gas porosities" (Eq. 10) are reported in Table 2. Water porosities are measured without confining pressure, whereas gas porosities are measured at three steps of a confining pressure cycle (2 $\mathrm{MPa}, 9 \mathrm{MPa}(\approx$ in-situ pressure) and $2 \mathrm{MPa}$ after unloading).

Gas porosities exhibit a low dispersion and range from 11.7 to $12.7 \%$ at $2 \mathrm{MPa}$ of confining pressure. Water porosities are more dispersed, mostly because of the presence of the two specimens MIL-20 and MIL-21 which have been sampled closer to the upper part of the received core than all other samples.

The water porosities appear to systematically underestimate the gas porosities by 1 to $2 \%$. As water molecule and Argon atom diameters are respectively $0.28 \mathrm{~nm}$ and $0.38 \mathrm{~nm}$, their size cannot explain this discrepancy.

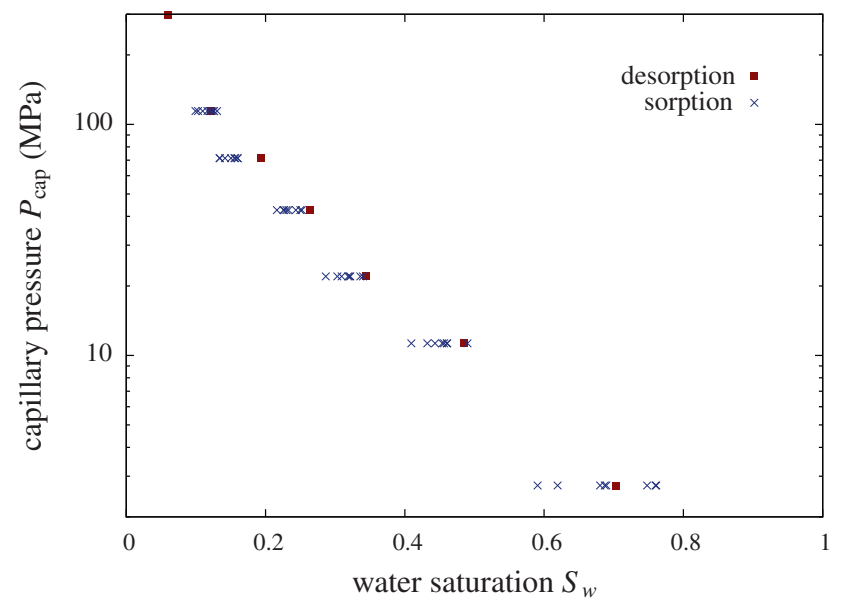

Figure 5

Capillary curve deduced from the $S_{w}-r h$ measurements (4) and Kelvin's law (1).

We rather believe that the water saturation method by immersion did not guarantee a total water saturation because of trapped gas. Another source of difference may be the sorption of Argon on pore walls during the porosity measurement experiment, which has not been quantitatively assessed.

The confining pressure increase from $2 \mathrm{MPa}$ to $9 \mathrm{MPa}$ leads to a decrease of $1 \%$ in porosity. After unloading, the initial porosity values are retrieved up to the measurement errors. This indicates that the evolution of the samples along the imposed stress path is mostly elastic.

\subsection{Capillary Curve}

In order to determine the length scales associated with the porosity, the water sorption and desorption curves are measured based on the method described in Section 1.2.1. The $S_{w}-r h$ curves obtained from relation (4) have been used to derive by means of Kelvin's law (1) the capillary pressure curves presented in Figure 5.

The desorption curve, which has been measured on a single sample, is close to the sorption curves, tending slightly towards higher water saturations. As for the set of eight sorption curves, the dispersion increases with decreasing capillary pressure $\left(S_{w}=0.68 \pm 0.10\right.$ at $P_{\text {cap }}=2.7 \mathrm{MPa}$; $S_{w}=0.115 \pm 0.015$ at $\left.P_{\text {cap }}=114 \mathrm{MPa}\right)$. Again, the two specimens MIL-20 and MIL-21, which have the highest total porosities, have also the weakest water saturation at $P_{\text {cap }}=2.7 \mathrm{MPa}$. This indicates that the extra porosity of these two specimens is mostly a macro-porosity.

The capillary pressure curves presented in Figure 5 are reinterpreted as cumulative pore size distributions in Figure 6 using Young-Laplace's law (2). To do so, the pores have 


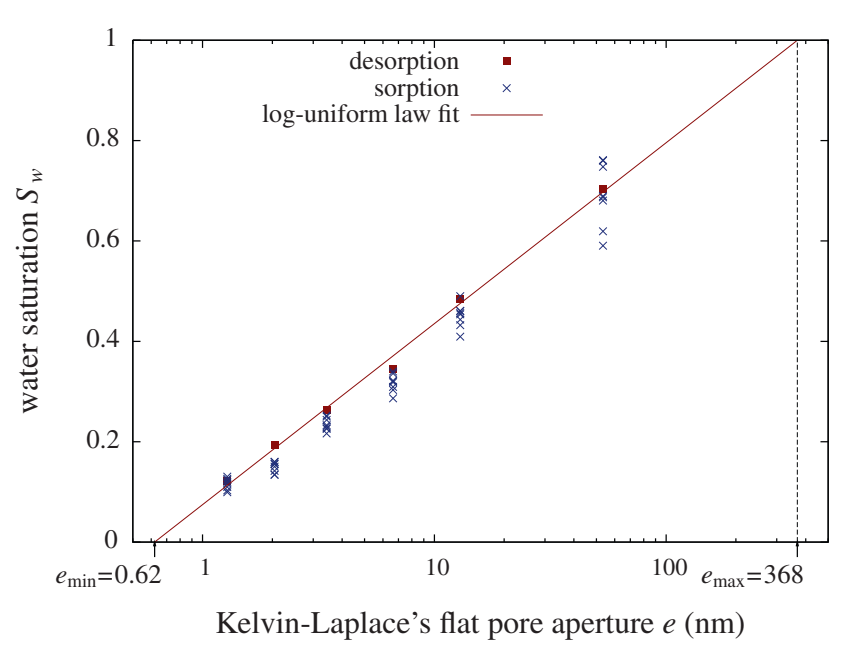

Figure 6

Pore aperture distribution according to Kelvin's (1) and YoungLaplace's (2) laws (for flat pores) and log-uniform law fit.

been assumed flat, i.e. described as the space between two parallel planes with an aperture $e$, which is of course a rough approximation.

Under these assumptions, around $70 \% \pm 5 \%$ of the porosity corresponds to pores with an aperture below $50 \mathrm{~nm}$. This fine porosity is attributed to the clay phase, which is located either in the canaliculi spanning carbonate aggregates or in the high-porosity regions (Fig. 2). The pore size distribution above $50 \mathrm{~nm}$ cannot be evaluated by the present method, but mainly corresponds to the inter-particular pores observed in the high-porosity regions.

Finally, in the studied range of pore sizes, a log-uniform repartition law provides an appropriate fit of the desorption curve. The minimum and maximum flat pore apertures of the fitted log-uniform law are respectively $0.6 \mathrm{~nm}$ and $370 \mathrm{~nm}$. As these extremal values are out of the range of validity of the present method (which is $\approx 2 \mathrm{~nm}$ to $50 \mathrm{~nm}$ ), they should be considered with care.

\subsection{Dry Gas Permeability}

The gas permeability has been initially measured on the dried samples using the steady state method presented in Section 1.2.2. Each sample undergoes the following confining pressure cycle: $2 \mathrm{MPa}, 9 \mathrm{MPa}$ and $2 \mathrm{MPa}$. At each confining pressure step, the apparent gas permeability is measured for three different gas injection pressures: $0.5 \mathrm{MPa}, 1 \mathrm{MPa}$ and $1.5 \mathrm{MPa}$. Except for the two specimens MIL-20 and MIL-21, the apparent gas permeability for an injection pressure of $1 \mathrm{MPa}$ is $1.25 \pm 0.15 \times 10^{-18} \mathrm{~m}^{2}$ and Klinkenberg corrected intrinsic permeability is
$2.4 \pm 1 \times 10^{-19} \mathrm{~m}^{2}$ (Tab. 3). The specimens MIL-20 and MIL-21, which also have more macro-porosity, have permeabilities 2 to 8 times greater than all other samples.

Reported Klinkenberg coefficients (except for specimen MIL-20) correspond to flat pore apertures in the order of 15 to $50 \mathrm{~nm}$ [11], according to a slip-flow model based on the kinetic theory of gases. These values are in excellent agreement with the estimated cumulative pore size distributions presented in Figure 6.

The moderate increase in confining pressure from 2 to $9 \mathrm{MPa}$ results in a decrease in permeability of only $10 \%$. After unloading to $2 \mathrm{MPa}$, the initial permeability is almost retrieved with less than $5 \%$ relative difference. As it is well known that small changes of the pore network can lead to significant changes in permeability, these relatively low fluctuations indicate that the deformation of the samples is mostly elastic during the present load cycle.

\subsection{Relative Gas Permeability}

In order to assess the relative gas permeability, effective gas permeability measurements are carried out on the partially water saturated samples. At each imposed relative humidity step, as in the dry case, the samples undergo a confining pressure cycle from $2 \mathrm{MPa}$ to $9 \mathrm{MPa}$ and back to $2 \mathrm{MPa}$. At each confining pressure step, the apparent effective gas permeability is measured for three different gas injection pressures: $0.5 \mathrm{MPa}, 1 \mathrm{MPa}$ and $1.5 \mathrm{MPa}$. For water saturations above 0.5 , Klinkenberg's law appeared inappropriate to model the effect of gas pressure. As a result, we choose to present relative gas permeabilities which correspond to apparent permeabilities (computed using Eq. 7) for an injection pressure of $1 \mathrm{MPa}$. The measured relative gas permeabilities are reported as a function of water saturation in Figure 7, for confining pressures of 2 and $9 \mathrm{MPa}$.

For water saturations below 0.5 , effective gas permeabilities exhibited limited hysteresis to a cycle in confining pressure (2-9-2 MPa). However at the highest water saturation tested (corresponding to $r h=98 \%$ ), the effective gas permeability after the confining pressure cycle ranged only from $1 / 2$ to $1 / 3$ of the value before the cycle, for the same reference value $(2 \mathrm{MPa})$ of confining pressure. Two hypotheses may be proposed to explain this hysteresis:

- pore compression due to hydrostatic loading induces a spatial reorganisation of the water or,

- the saturation cycle has induced micro-cracking of the samples.

Subsequent permeability measurements after a second drying of the samples suggest that limited micro-cracking might indeed have occurred.

Excepted for the two outlier specimens MIL-20 and MIL-21 which suffered from severe micro-cracking induced by the saturation process, all the measured relative 
TABLE 3

Gas permeability in the dry state

\begin{tabular}{|c|c|c|c|c|c|c|}
\hline \multirow[b]{3}{*}{ Sample } & \multicolumn{6}{|c|}{ Confining pressure (MPa) } \\
\hline & 2 & 9 & 2 & 9 & 2 & 9 \\
\hline & \multicolumn{2}{|c|}{$K^{\mathrm{app}}\left(P_{i}=1 \mathrm{MPa}\right)\left(\mathrm{m}^{2}\right)$} & \multicolumn{2}{|c|}{$K^{\mathrm{int}}\left(\mathrm{m}^{2}\right)$} & \multicolumn{2}{|c|}{$\beta(\mathrm{MPa})$} \\
\hline MIL-12 & $1.44 \times 10^{-18}$ & $1.28 \times 10^{-18}$ & $2.8 \times 10^{-19}$ & $2.3 \times 10^{-19}$ & 2.4 & 2.8 \\
\hline MIL-13 & $1.20 \times 10^{-18}$ & $1.12 \times 10^{-18}$ & $1.8 \times 10^{-19}$ & $1.6 \times 10^{-19}$ & 3.4 & 3.6 \\
\hline MIL-14 & $1.21 \times 10^{-18}$ & $1.13 \times 10^{-18}$ & $1.9 \times 10^{-19}$ & $1.8 \times 10^{-19}$ & 3.3 & 3.2 \\
\hline MIL-20 & $9.41 \times 10^{-18}$ & $3.94 \times 10^{-18}$ & $7.0 \times 10^{-18}$ & $2.4 \times 10^{-18}$ & 0.23 & 0.40 \\
\hline MIL-21 & $2.15 \times 10^{-18}$ & $1.83 \times 10^{-18}$ & $5.5 \times 10^{-19}$ & $4.4 \times 10^{-19}$ & 1.7 & 1.9 \\
\hline MIL-22 & $1.36 \times 10^{-18}$ & $1.26 \times 10^{-18}$ & $4.2 \times 10^{-19}$ & $3.8 \times 10^{-19}$ & 1.1 & 1.0 \\
\hline MIL-23 & $1.13 \times 10^{-18}$ & $1.05 \times 10^{-18}$ & $1.8 \times 10^{-19}$ & $1.6 \times 10^{-19}$ & 3.3 & 3.3 \\
\hline MIL-24 & $1.16 \times 10^{-18}$ & $1.09 \times 10^{-18}$ & $1.9 \times 10^{-19}$ & $2.1 \times 10^{-19}$ & 3 & 2.5 \\
\hline
\end{tabular}

permeabilities in Figure 7 lie on a master curve, for both confining pressures ( 2 and $9 \mathrm{MPa})$. The main feature of the relative permeability curve is the presence of a low critical water saturation $S_{w, c}$ around $0.71 \pm 0.04$ for which gas permeability nearly vanishes. Such so-called hydraulic cutoff has also been observed on tight sandstones [5].

The influence of the saturation history on the relative permeability curve has not been assessed and requires further investigation. This effect has been studied on two industrial concretes in [4], comparing desorption and re-saturation paths. It has been observed for these concretes that the saturation history influences relative gas permeability mainly in the case of a strong hysteresis in desorption/re-saturation isotherms (i.e. $S_{w}-r h$ relations). The higher relative gas permeability for the re-saturation path has been attributed to bottle neck effects within the pore network or capillary pressure effects. However, as the imbibition and drainage capillary curves (Fig. 5) do not significantly differ, one may expect little influence of the saturation history on the relative gas permeability for the material presently under consideration.

\section{MULTI-SCALE MODELING}

In this section, a method to estimate the relative gas permeability of the present tight carbonate rock with clay content is proposed. We assume the existence of a Representative Volume Element (RVE) for the permeability of this porous material. Two distinct scales are hence considered:

- the macroscopic scale, which is supposed to correspond to the scale at which the previous experiments have been carried out;
- the microscopic scale at which one may distinguish elementary particles such as carbonate aggregates, clay particles and pores.

The upscaling is performed within the framework of random media or so-called continuum micromechanics [12], widely used to derive effective mechanical properties. This strategy has been applied to determine the effective permeability of specific porous media such as fractured media [13-17], argillite [18] or tight sandstones with inter-granular hydraulic joints [19]. Due to the disordered or polycrystalline-like arrangement of the elementary particles at the pore scale, the self-consistent homogenisation scheme initially proposed by [20] in linear elasticity has been extensively used to estimate the macroscopic permeability tensor $K^{\text {hom }}$. The self-consistent scheme naturally exhibits a so-called percolation threshold in the case of a strong contrast in permeability of the elementary particles. We wish to take advantage of this feature to model the hydraulic cut-off which has been observed experimentally at the critical water saturation.

\subsection{Description of the Microscopic Scale}

A direct way to upscale the permeability would be to resort to numerical homogenisation on explicit representations of the microstructure, either obtained by 3D imaging techniques or computer synthesised [21-28]. We instead turn to analytical modeling with an implicit description of the microstructure.

Our first modeling step lies in the extraction of the most salient properties of the organisation of the microstructure. We purposely wish to avoid a too exhaustive description as it would lead to a model with too many parameters, 


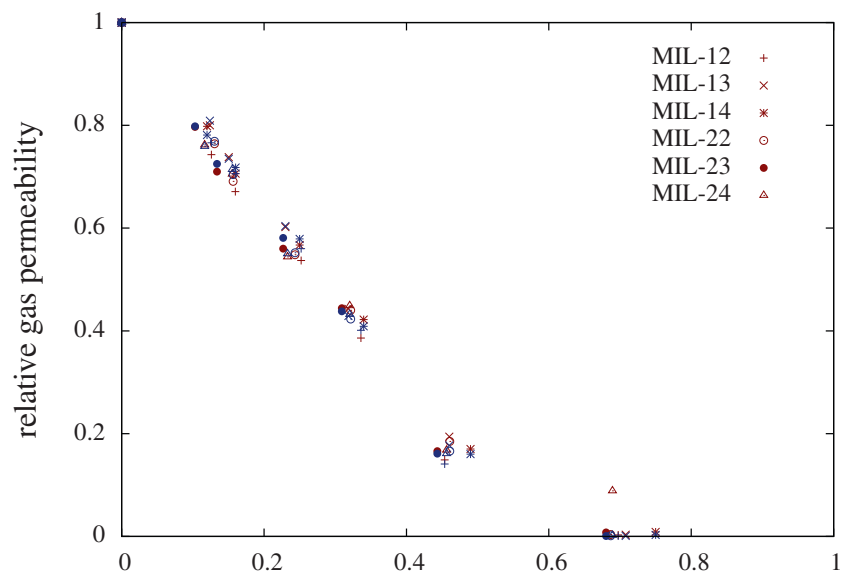

a)

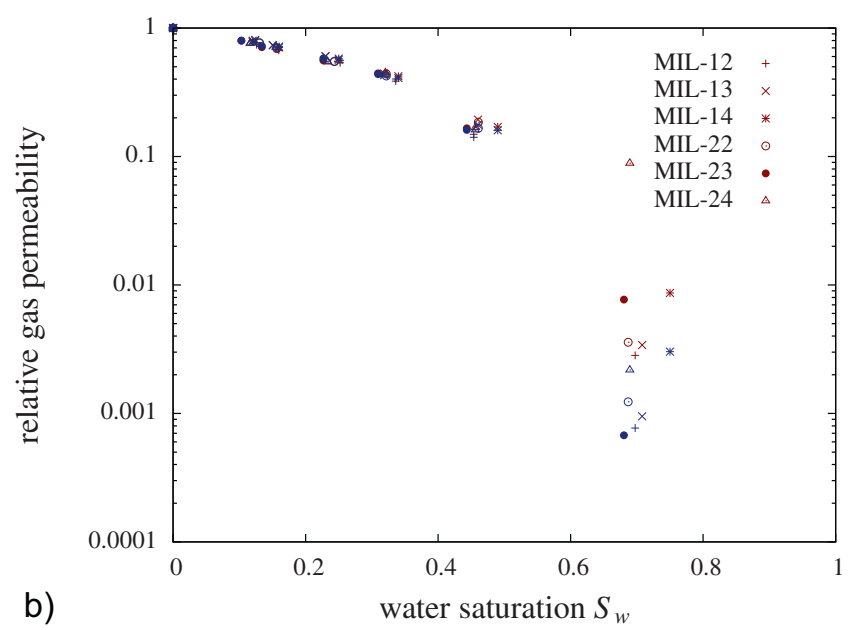

Figure 7

Measured relative gas permeabilities at confining pressures of $2 \mathrm{MPa}$ (red) and $9 \mathrm{MPa}$ (blue). a) Normal scale; b) log-scale.

thus of limited practical use. From a hydraulic point of view, the pore structure may be simplified as follows (Fig. 2): most of the pores are concentrated in high-porosity regions together with clay particles. These regions of micro-metric extent $(\approx 2 \mu \mathrm{m})$ lie in between larger carbonate aggregates or silts (10 to $20 \mu \mathrm{m}$ ), which are assumed too dense to be significantly permeable. The key point is that the connection between the high-porosity regions is only ensured by canaliculi (resp. joints) at the outskirt of the large carbonate aggregates (resp. silts). In other words, the flow is assumed to be mostly controlled by these canaliculi and joints, while the high-porosity regions play a minor role.

A $\operatorname{RVE} \Omega$ of volume $|\Omega|$ of this material is now considered. The high-porosity regions occupy the domain $\Omega_{h p}$ with volume fraction $f=\left|\Omega_{h p}\right| /|\Omega|$. To simplify the discussion,

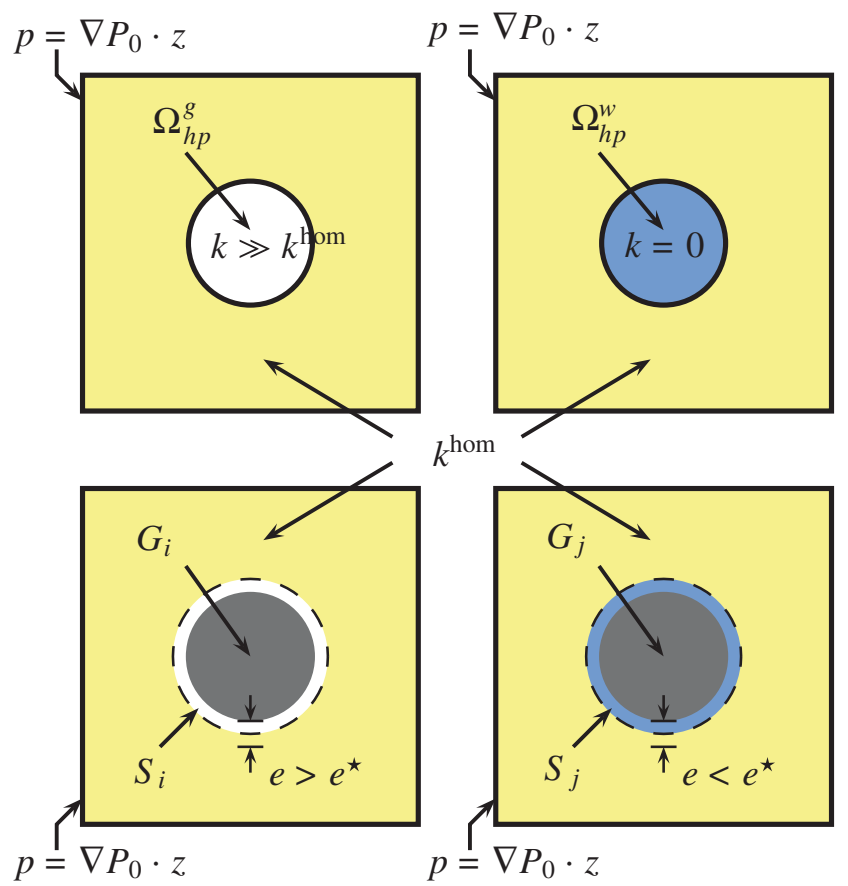

Figure 8

Generalised Eshelby problems involved in the self-consistent homogenisation scheme. $G_{j}\left(e<e^{\star}\right)$ : grain $j$ surrounded by a half interface $S_{j}\left(e<e^{\star}\right)$ filled with water, $G_{i}\left(e>e^{\star}\right)$ : grain $i$ surrounded by a half interface $S_{i}\left(e>e^{\star}\right)$ filled with gas, $\Omega_{h p}^{w}$ : water saturated high porosity region, $\Omega_{h p}^{g}$ : gas saturated high porosity region.

carbonate aggregates surrounded by canaliculi and silts with hydraulic joints will be generically called grains with (hydraulic) interfaces. Each interface, which may contain several clay particles, is assumed to have an equivalent aperture $e$ for the fluid flow. To describe the grain-interface phases (Fig. 8), the composite elementary particle of interest is a grain surrounded by an interface (more precisely, just a half-interface as will be explained later). Let $G_{i}(e)$ be the domain occupied by a grain $i$, such that its surrounding hydraulic interface has an actual aperture $e$. The boundary $S_{i}(e)$ of each grain $i$ corresponds to a half interface. The total domain occupied by all the grains is denoted $\Omega_{g}=\cup_{i} G_{i}$. The volume fraction of all the grains is then $1-f$. As the interfaces are very thin as compared to the radius of the grains, their contribution to the pore volume is neglected.

The apertures $e$ of the interfaces are not all equal; instead, they are assumed to follow a distribution $\alpha(e)$ in the range [ $\left.e_{\min }, e_{\max }\right]$ where $e_{\min }$ and $e_{\max }$ are respectively the smallest and the largest interface apertures. The distribution is normalised such that $\int_{e_{\min }}^{e_{\max }} \alpha(e) \mathrm{d} e=1$. The description of the pore space based on size distribution has been classically 
used in statistical modes based on bundles of cylindrical pores [29-32].

In the case of a partial water saturation $S_{w}$ resulting from an imposed capillary pressure $P_{\text {cap }}$, we denote $e^{\star}$ the interface aperture which corresponds to $P_{\text {cap }}$ via Young-Laplace's law. All the interfaces with apertures $e<e^{\star}$ are assumed fully water saturated, while all interfaces with apertures $e>e^{\star}$ are assumed fully gas saturated. It is then convenient to introduce the fraction $s \in[0,1]$ of water saturated interfaces, which is related to the interface aperture distribution by:

$$
s=\int_{e_{\min }}^{e^{\star}} \alpha(e) \mathrm{d} e
$$

\subsection{Hydraulic Properties of the Phases}

In the framework of periodic homogenisation, [33] has shown that the steady state, laminar, single phase flow of an incompressible Newtonian viscous fluid in a porous medium obeys Darcy's law at the macroscopic scale. However, the direct resolution of the Stokes equations on a true RVE of the microstructure is a difficult task. First, the very disperse pore sizes involved and the complexity of their connections make it almost impossible to obtain a well defined, truly representative element of the pore network of an actual rock such as considered in this paper - despite all the advanced imaging techniques currently available. Further, in the case where such RVE were available, it would be far too computationally intensive to solve Stokes equations with a reasonable resolution.

This has motivated authors to propose simplifications of the problem by replacing the different constituents of the micro-scale such as micro-cracks [13-17], inter-granular joints [19] or flat pores [18] by fictitious Darcy media. The simplified problem then amounts to the homogenisation of a heterogeneous Darcy medium. Based on these considerations, let us propose the following fictitious equivalent permeabilities of the elementary particles of each phase.

\subsubsection{Dry Case}

To start with, let us consider the case where all pores are gas saturated. In what follows, we systematically consider a gas viscosity $\mu=1$, with no loss of generality.

\section{High-Porosity Regions}

At the micro-metric scale, an isotropic apparent permeability $\boldsymbol{K}_{h p}=K_{h p} \mathbf{1}$ (where $\mathbf{1}$ is the second order identity tensor) is attributed to the high-porosity regions which occupy the domain $\Omega_{h p}$. It is assumed that this permeability could be obtained from the upscaling of a Stokes flow on a representative volume of the high-porosity region from a lower scale. Dimensional analysis indicates that $K_{h p}$ scales as the square of a characteristic pore size. As will be seen later, further dimensional analysis will indicate that this scaling for $K_{h p}$ is much greater than the effective permeability $K^{\text {hom }}$ of the RVE, so that exact value of $K_{h p}$ is not needed in a first approximation. Physically speaking, this corresponds to the case where the pressure drop in the high-porosity regions can be neglected as compared to the pressure drop along the interfaces.

\section{Grains with Interfaces}

As proposed for tight sandstones in [19], a two dimensional mathematical representation of the interfaces will be adopted. This is justified by the very thin openings $e$ of the canaliculi or of the joints ( $e$ smaller than a few hundreds of nanometres, generally much smaller) as compared to the grain radii $R$ which are around $10 \mu \mathrm{m}$ (i.e. $e \ll R$ ). At the micro-metric scale, the core of a grain $G_{i}$ is assumed impermeable $(K \rightarrow 0)$. In turn, based on Poiseuille's flow between two planes with aperture $e$, the flow in the half interface $S_{i}(e)$ surrounding the grain $G_{i}(e)$ is described by a surface Darcy's law:

$$
\boldsymbol{q}(\boldsymbol{z})=-\eta(e) \operatorname{grad}_{s} p(\boldsymbol{z}) \quad\left(\boldsymbol{z} \in S_{i}(e)\right)
$$

where $p(z)$ is the pressure at a point $z$ of the interface, $\eta$ is a surface permeability and $\boldsymbol{q}(\boldsymbol{z})$ is the surface velocity vector defined as the cumulative velocity across the half interface:

$$
\boldsymbol{q}(\boldsymbol{z})=\int_{0}^{e / 2} \boldsymbol{v}(\boldsymbol{z}+Z \boldsymbol{n}) \mathrm{d} Z
$$

where $\boldsymbol{n}$ is the unit external normal to the boundary of the grain at point $z$. The reason to consider at this step only one half of the interface surrounding a grain $G_{i}(e)$ is to avoid double counting in the estimation of the total flow through all the interfaces, which will later be retrieved by summation over all grains.

The classical solution to Poiseuille's flow between two planes with aperture $e$ and the definition of the surface velocity (Eq. 13) lead to the definition of the surface permeability in the law in Equation (12) as:

$$
\eta(e)=\frac{e^{3}}{24}
$$

for a half interface [19].

\subsubsection{Partially Saturated Case}

In the case of a partial water saturation $S_{w}$ of the pore network, we assume that the water does not move due to gas 
flow. This decoupling in the extension of Darcy's law to twophase flow is frequently assumed in the capillary dominated flow regime (i.e. when viscous forces are negligible in comparison to capillary forces) [2]. It is here justified on a macroscopic level by the above presented experiments in which gas flow did not significantly modify the overall water saturation of the samples.

The interfaces which are water saturated are assumed to have a vanishing gas permeability. Consequently, the grains surrounded by water saturated interfaces behave as fully gas impermeable particles.

Further, it is assumed for simplification that a subdomain $\Omega_{h p}^{w} \subset \Omega_{h p}$ of volume fraction $f S_{w}$ of highporosity regions is totally water saturated and impermeable to gas (Fig. 8). The complementary subdomain $\Omega_{h p}^{g} \subset \Omega_{h p}$ of volume fraction $f\left(1-S_{w}\right)$ is assumed fully gas saturated, with unchanged gas permeability. These are strong assumptions which may have to be reconsidered in future works.

\subsection{Determination of the Macroscopic Permeability by the Self Consistent Estimate}

\subsubsection{Darcy Medium Homogenisation Problem}

Due to the previous simplifications consisting of replacing elementary particles by fictitious Darcy media, we are left with the homogenisation of a heterogeneous Darcy media. The homogenisation problem is defined on the RVE $\Omega$ by:

$$
\begin{array}{ll}
\operatorname{div} \boldsymbol{v}=0 & (\boldsymbol{z} \in \Omega) \\
\boldsymbol{v}=-\boldsymbol{K}_{h p} \cdot \operatorname{grad} p & \left(\boldsymbol{z} \in \Omega_{h p}^{g}\right) \\
\boldsymbol{v}=0 & \left(\boldsymbol{z} \in \Omega_{h p}^{w} \cup \Omega_{g}\right) \\
\boldsymbol{q}=-\eta(e) \operatorname{grad}_{s} p & \left(\boldsymbol{z} \in S_{i}(e), e>e^{\star}\right) \\
\boldsymbol{q}=0 & \left(\boldsymbol{z} \in S_{i}(e), e<e^{\star}\right) \\
p=\nabla P \cdot \boldsymbol{z} & (\boldsymbol{z} \in \partial \Omega)
\end{array}
$$

where $z$ is the position vector, $\nabla P$ is the macroscopic pressure gradient which is applied uniformly at the boundary $\partial \Omega$ of the RVE and $\boldsymbol{q}$ is the cumulative velocity across the half interface defined by Equation (13).

A direct consequence of the uniform pressure gradient boundary condition is the so-called pressure gradient averaging rule, which relates the local pressure gradients to the macroscopic pressure gradient by:

$$
\nabla P=\frac{1}{|\Omega|} \int_{\Omega} \operatorname{grad} p \mathrm{~d} V \equiv \overline{\operatorname{grad} p}
$$

Decomposition of the volume average over the elementary particles yields:

$$
\begin{aligned}
\nabla P= & f\left(1-S_{w}\right) \overline{\operatorname{\operatorname {grad}} p^{h p, g}}+f S_{w} \overline{\operatorname{grad} p^{h p, w}} \\
& +(1-f) \int_{e_{\min }}^{e^{\star}} \overline{\operatorname{grad} p^{G_{i}}(e)} \alpha(e) \mathrm{d} e \\
& +(1-f) \int_{e^{\star}}^{e_{\max }} \overline{\operatorname{grad} p^{G_{i}}(e)} \alpha(e) \mathrm{d} e
\end{aligned}
$$

where the notation:

$$
\overline{\operatorname{grad} p} p^{h p, w}=\frac{1}{\left|\Omega_{h p}^{w}\right|} \int_{\Omega_{h p}^{w}} \operatorname{grad} p \mathrm{~d} V
$$

indicates the intrinsic volume average over the phase $\Omega_{h p}^{w}$, and similarly for the phase $\Omega_{h p}^{g}$ or the grains $G_{i}(e)$.

In turn, the macroscopic velocity $\boldsymbol{V}$ corresponds to the volume average of the local velocity. By linearity of the homogenisation problem (15), $\boldsymbol{V}$ is linearly related to the macroscopic pressure gradient by the macroscopic permeability $\boldsymbol{K}^{\text {hom: }}$

$$
\boldsymbol{V}=\overline{\boldsymbol{v}}=-\boldsymbol{K}^{\mathrm{hom}} \cdot \nabla P
$$

The overall volume average of the velocity can be decomposed on phases and grains similarly to Equation (17). Hence, the macroscopic gas permeability $\boldsymbol{K}^{\text {hom }}$ can be deduced from the knowledge of the phase averages (or elementary particles averages) of the pressure gradient and velocity.

\subsubsection{Generalised Eshelby Problems}

The self-consistent scheme relies on the estimate of the velocity and pressure gradient phase averages involved (e.g. in Eq. 17 and Eq. 19) based on the solution to auxiliary problems: the so-called Eshelby problems.

The auxiliary problems are devised as follows. For each phase, an inclusion $I$ (or elementary particle representative of the phase) is embedded in an infinite medium (Fig. 8). The infinite medium has a uniform permeability $\boldsymbol{K}_{0}$. An auxiliary uniform pressure gradient $\nabla P_{0}$ is applied at infinity (remote boundary conditions). The resolution of this problem yields the average of the pressure gradient and of the velocity in the inclusion linearly as a function of the auxiliary pressure gradient $\nabla P_{0}$. If the inclusion $I$ is an ellipsoid with homogeneous permeability $\boldsymbol{K}_{I}$, the pressure gradient and the velocity are actually constant inside the inclusion. In this case, the pressure gradient inside the inclusion is given by:

$$
\operatorname{grad} p(\boldsymbol{z})=\left[\mathbf{1}+\boldsymbol{P}_{0}^{I} \cdot\left(\boldsymbol{K}_{I}-\boldsymbol{K}_{0}\right)\right]^{-1} \cdot \nabla P_{0} \quad(\boldsymbol{z} \in I)
$$

where $\boldsymbol{P}_{0}^{I}$ is the so-called Hill tensor of the inclusion $I$ in the infinite medium of permeability $\boldsymbol{K}_{0}$, which is related to the 
Eshelby tensor $\boldsymbol{S}_{0}^{I}$ by $\boldsymbol{S}_{0}^{I}=\boldsymbol{P}_{0}^{I} \cdot \boldsymbol{K}_{0}$. In the case where $\boldsymbol{K}_{0}$ is isotropic (i.e. $\boldsymbol{K}_{0}=K_{0} \mathbf{1}$ ), the Eshelby tensor is independent of $K_{0}$. For a spherical or spheroidal (oblate or prolate) inclusion $I$, the Eshelby tensor decomposes as $\boldsymbol{S}=S_{n} \boldsymbol{n} \otimes \boldsymbol{n}+S_{t}(\mathbf{1}-\boldsymbol{n} \otimes \boldsymbol{n})$ [1], with:

$$
\begin{aligned}
\boldsymbol{S}^{\text {sphere }}= & \frac{\mathbf{1}}{3} \\
\boldsymbol{S}^{\text {prolate }}= & \frac{-\sqrt{\omega^{2}-1}+\omega \operatorname{arccosh}(\omega)}{\left(\omega^{2}-1\right)^{3 / 2}} \boldsymbol{n} \otimes \boldsymbol{n} \\
& +\frac{\omega\left(\omega \sqrt{\omega^{2}-1}-\operatorname{arccosh}(\omega)\right)}{2\left(\omega^{2}-1\right)^{3 / 2}}(\mathbf{1}-\boldsymbol{n} \otimes \boldsymbol{n}) \\
\boldsymbol{S}^{\text {oblate }}= & -\frac{-\sqrt{1-\omega^{2}}+\omega \arccos (\omega)}{\left(1-\omega^{2}\right)^{3 / 2}} \boldsymbol{n} \otimes \boldsymbol{n} \\
& -\frac{\omega\left(\omega \sqrt{1-\omega^{2}}-\arccos (\omega)\right)}{2\left(1-\omega^{2}\right)^{3 / 2}}(\mathbf{1}-\boldsymbol{n} \otimes \boldsymbol{n})
\end{aligned}
$$

where $\omega$ is the aspect ratio of the spheroid $(\omega>1$, prolate; $\omega<1$, oblate) and $\boldsymbol{n}$ is the unit vector collinear with the axis of revolution of the spheroid. The velocity inside the inclusion is then simply deduced from the combination of Darcy's law with the Eshelby localisation rule (20) as $\boldsymbol{v}(\boldsymbol{z})=-\boldsymbol{K}_{I} \cdot \operatorname{grad} p(\boldsymbol{z})$.

The case of a generalised Eshelby problem with a composite inclusion made of an impermeable core surrounded by a permeable interface (in the sense of Eq. 12) as been dealt with by [19]. A remarkable result is that the intrinsic average of the pressure gradient and of the velocity in the composite inclusion (i.e. including the interface) can be retrieved from Equation (20) in the uniform spherical inclusion case for an appropriate choice of equivalent fictitious uniform permeability $\boldsymbol{K}^{e q}=K^{e q} \mathbf{1}$ of the inclusion. If $\eta$ denotes the surface permeability (Eq. 12) and $R$ the radius of the composite inclusion, this equivalent fictitious permeability is:

$$
K^{e q}=\frac{2 \eta}{R}
$$

Another remarkable result is that $\boldsymbol{K}^{e q}$ does not depend on the permeability $\boldsymbol{K}_{0}$ of the surrounding medium. This last feature is specific to a Darcy medium as it is untrue for linear elasticity (see e.g. [34], Eq. 37). Moreover, this equivalent permeability corresponds to the limiting case of a composite sphere made of a rigid core and a fluid shell of vanishing thickness in which an incompressible Newtonian fluid flows, with uniform normal velocity/no tangential stress boundary conditions on the outside of the composite sphere $[11,35,36]$.

Alternatively, the result in Equation (22) can be retrieved from a generalised Eshelby problem with a composite spherical inclusion made of an impermeable core surrounded by a shell with thickness $h$ and isotropic permeability $\eta / h$ in the limit as $h \rightarrow 0$. Furthermore, the resolution of a generalised Eshelby problem with a composite inclusion made of an impermeable spheroidal core surrounded by a confocal spheroidal shell with isotropic permeability $\eta / h$ and vanishing average thickness $h$ indicates that an equivalent transverse isotropic permeability tensor independent of $\boldsymbol{K}_{0}$ can be similarly defined.

\subsubsection{Self-Consistent Estimate}

The principle of the self-consistent estimate is to choose the permeability $\boldsymbol{K}_{0}$ of the infinite medium in the Eshelby problems equal to the homogenised permeability $\boldsymbol{K}^{\text {hom }}$. In turn, the auxiliary pressure gradient $\nabla P_{0}$ in the Eshelby problem is determined as a linear function of the actual macroscopic pressure gradient $\nabla P$ by inserting the Eshelby localisation rule of the pressure gradient in Equation (20) in the pressure gradient averaging rule (17). Combination with Equation (19) classically leads to an implicit equation on the self-consistent estimate of the macroscopic permeability $\boldsymbol{K}^{\text {hom }}$ :

$$
\boldsymbol{K}^{\mathrm{hom}}=\overline{\boldsymbol{K} \cdot\left[\mathbf{1}+\boldsymbol{P}_{\text {hom }}^{I} \cdot \delta \boldsymbol{K}\right]^{-1}} \cdot\left(\overline{\left[\mathbf{1}+\boldsymbol{P}_{\text {hom }}^{I} \cdot \delta \boldsymbol{K}\right]^{-1}}\right)^{-1}
$$

where $\delta \boldsymbol{K}=\boldsymbol{K}-\boldsymbol{K}^{\text {hom }}$. Equation (23) can be simplified into:

$$
\overline{\delta \boldsymbol{K} \cdot\left[\mathbf{1}+\boldsymbol{P}_{\mathrm{hom}}^{I} \cdot \delta \boldsymbol{K}\right]^{-1}}=\mathbf{0}
$$

In the following developments, all the grains are assumed spheroidal with the same aspect ratio $\omega$. The distribution of the orientations of the spheroidal grains is assumed isotropic. Based on the results recalled in the previous subsection, an equivalent gas permeability tensor $\boldsymbol{K}^{e q}(e)$ is associated with grains surrounded by an interface of thickness $e>e^{\star}$ whereas the grains with water saturated interfaces are gas-impermeable. Denoting the unit vector collinear with the axis of revolution of the spheroid by $\boldsymbol{n}$, the transverse isotropic equivalent permeability tensor decomposes as $\boldsymbol{K}^{\mathrm{eq}}(e)=K_{n}^{\mathrm{eq}}(e) \boldsymbol{n} \otimes \boldsymbol{n}+K_{t}^{\mathrm{eq}}(e)(\mathbf{1}-\boldsymbol{n} \otimes \boldsymbol{n})$. The Eshelby and Hill tensors of the grains are respectively denoted $\boldsymbol{S}_{\text {hom }}^{g}(\omega)$ and $\boldsymbol{P}_{\text {hom }}^{g}(\omega)$. In turn, the high porosity regions are described by spherical inclusions with gas permeability $\boldsymbol{K}^{\text {hp }}$ when they are gas saturated or 0 when they are water saturated. The Eshelby and Hill tensors of the spherical high porosity regions are respectively denoted $\boldsymbol{S}_{\text {hom }}^{h p}$ and $\boldsymbol{P}_{\text {hom }}^{h p}$. Consequently, the volume 
average in Equation (24) can be split as in Equation (17) into:

$$
\begin{aligned}
& -f S_{w} \boldsymbol{K}^{\mathrm{hom}} \cdot\left[\mathbf{1}-\boldsymbol{S}_{\mathrm{hom}}^{h p}\right]^{-1} \\
& +f\left(1-S_{w}\right)\left(\boldsymbol{K}^{\mathrm{hp}}-\boldsymbol{K}^{\mathrm{hom}}\right) \cdot\left[\mathbf{1}+\boldsymbol{P}_{\mathrm{hom}}^{h p} \cdot\left(\boldsymbol{K}^{\mathrm{hp}}-\boldsymbol{K}^{\mathrm{hom}}\right)\right]^{-1} \\
& -(1-f) \int_{e^{\min } e^{\star}}<\boldsymbol{K}^{\mathrm{hom}} \cdot\left[\mathbf{1}-\boldsymbol{S}_{\mathrm{hom}}^{g}(\omega)\right]^{-1}>\alpha(e) \mathrm{d} e \\
& +(1-f) \int_{e^{\star}}^{e^{\max }}<\left(\boldsymbol{K}^{\mathrm{eq}}(e)-\boldsymbol{K}^{\mathrm{hom}}\right) \\
& \quad\left[\mathbf{1}+\boldsymbol{P}_{\mathrm{hom}}^{g} \cdot\left(\boldsymbol{K}^{\mathrm{eq}}(e)-\boldsymbol{K}^{\mathrm{hom}}\right)\right]^{-1}>\alpha(e) \mathrm{d} e=\mathbf{0}
\end{aligned}
$$

where $\langle\ldots\rangle$ indicates the angular average over all directions of the orientations of the spheroids.

Under the hypothesis of isotropy, the macroscopic permeability is $\boldsymbol{K}^{\text {hom }}=K^{\text {hom }} \mathbf{1}$ and Equation (24) reduces to a single scalar equation. Making use of:

- the orthogonality properties of the tensors $\boldsymbol{n} \otimes \boldsymbol{n}$ and $\mathbf{1}-\boldsymbol{n} \otimes \boldsymbol{n}$,

- the angular average of $\boldsymbol{n} \otimes \boldsymbol{n}$ for an isotropic distribution of orientations $<n \otimes n>=1 / 3$,

- the definition of the fraction $s$ of water saturated interfaces (11) allows simplification of Equation (25) to:

$$
\begin{aligned}
& -f S_{w} \frac{3}{2}+f\left(1-S_{w}\right) 3 \frac{K^{\mathrm{hp}} / K^{\mathrm{hom}}-1}{2+K^{\mathrm{hp}} / K^{\mathrm{hom}}} \\
& -(1-f) s \frac{1}{3}\left[\frac{1}{1-S_{n}(\omega)}+\frac{2}{1-S_{t}(\omega)}\right] \\
& +(1-f) \frac{1}{3} \int_{e^{\star}}^{e^{\max }}\left[\frac{K_{n}^{\mathrm{eq}}(e) / K^{\mathrm{hom}}-1}{1+S_{n}(\omega)\left(K_{n}^{\mathrm{eq}}(e) / K^{\mathrm{hom}}-1\right)}\right. \\
& \left.+2 \frac{K_{t}^{\mathrm{eq}}(e) / K^{\mathrm{hom}}-1}{1+S_{t}(\omega)\left(K_{t}^{\mathrm{eq}}(e) / K^{\mathrm{hom}}-1\right)}\right] \alpha(e) d e=0
\end{aligned}
$$

The percolation threshold of the self-consistent scheme is reached for the critical water saturation $S_{w}^{c}$ and interface water saturation $s^{c}$ such that $K^{\text {hom }} \rightarrow 0$. Inserting this limiting case in Equation (26) indicates that the critical water saturations for the present model verify:

$$
\begin{gathered}
3 f\left[1-\frac{3}{2} S_{w}^{c}\right]+\frac{1-f}{3}\left[\left(1-s^{c}\right)\left(\frac{1}{S_{n}(\omega)}+\frac{2}{S_{t}(\omega)}\right)\right. \\
\left.-s^{c}\left(\frac{1}{1-S_{n}(\omega)}+\frac{2}{1-S_{t}(\omega)}\right)\right]=0
\end{gathered}
$$

irrespective of the values of $K^{\mathrm{hp}}$ and $\boldsymbol{K}^{\mathrm{eq}}(e)$, as long as they are not null. The influence of the grains aspect ratio $\omega$ and of the high porosity regions volume fraction $f$ is illustrated in Figure 9 under the assumption that the interface water saturation $s$ is equal to the total water saturation $S_{w}$.

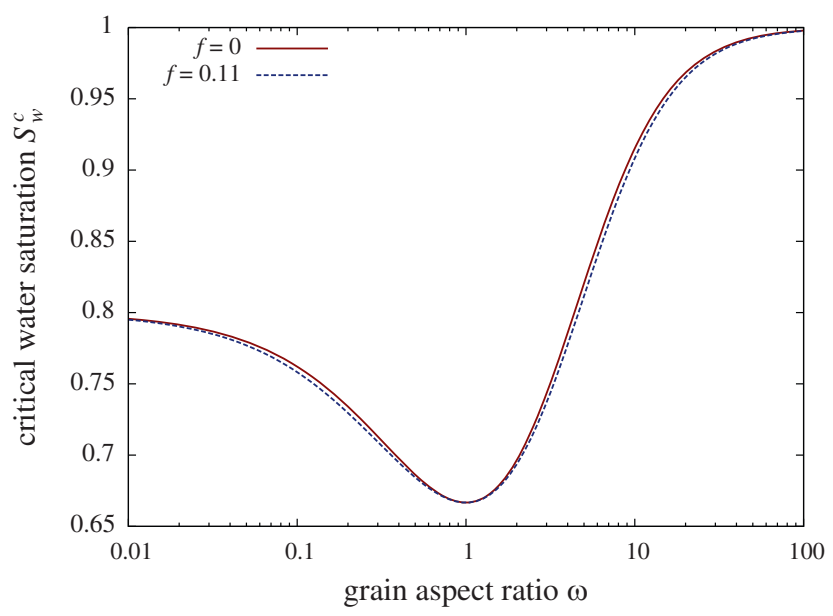

Figure 9

Critical water saturation for which the gas permeability vanishes in the case $S_{w}=s$.

In order to provide an explicit expression of the relative gas permeability, we choose to use a spherical shape of the inclusions in the Eshelby problems. The grains are assumed to all have the same radius $R$. Further, the experimental results presented in Figure 6 hint that a log-uniform distribution of interface apertures is a satisfactory approximation, that is:

$$
\alpha(e)=\frac{1}{\ln \left(e_{\max } / e_{\min }\right)} \times \frac{1}{e}
$$

For the log-uniform distribution, the median interface aperture $e_{\text {med }}$, the maximum to minimum aperture ratio $\rho$ and the critical aperture $e^{\star}$ which separates water saturated from gas saturated interfaces are:

$$
\begin{aligned}
e_{\mathrm{med}} & =\sqrt{e_{\min } e_{\max }} \\
\rho & =e_{\max } / e_{\min } \\
e^{\star} & =e_{\min }^{1-s} e_{\max }^{s}=e_{\max } \rho^{s-1}
\end{aligned}
$$

In the case where the high porosity region permeability $K^{h p} \gg K^{\text {hom }}$, the solution to equation (26) is then explicit:

$$
K^{\mathrm{hom}}=\frac{e_{\mathrm{med}}^{3}}{24 R} \frac{\rho^{\frac{3}{2}}-\rho^{\frac{1-3 f\left(1-S_{w}\right)}{1-f}(2 s-1)}}{\rho^{\frac{1-3 f\left(1-S_{w}\right)}{1-f}}-1}
$$

The approximation $K^{h p} \gg K^{\text {hom }}$ can be a posteriori justified by the fact that the high porosity region permeability scales as the square of a characteristic pore size $\left(K^{h p}=\mathcal{O}\left(e_{\text {med }}^{2}\right)\right)$ whereas the homogenised permeability $K^{\text {hom }}$ (Eq. 30), like 

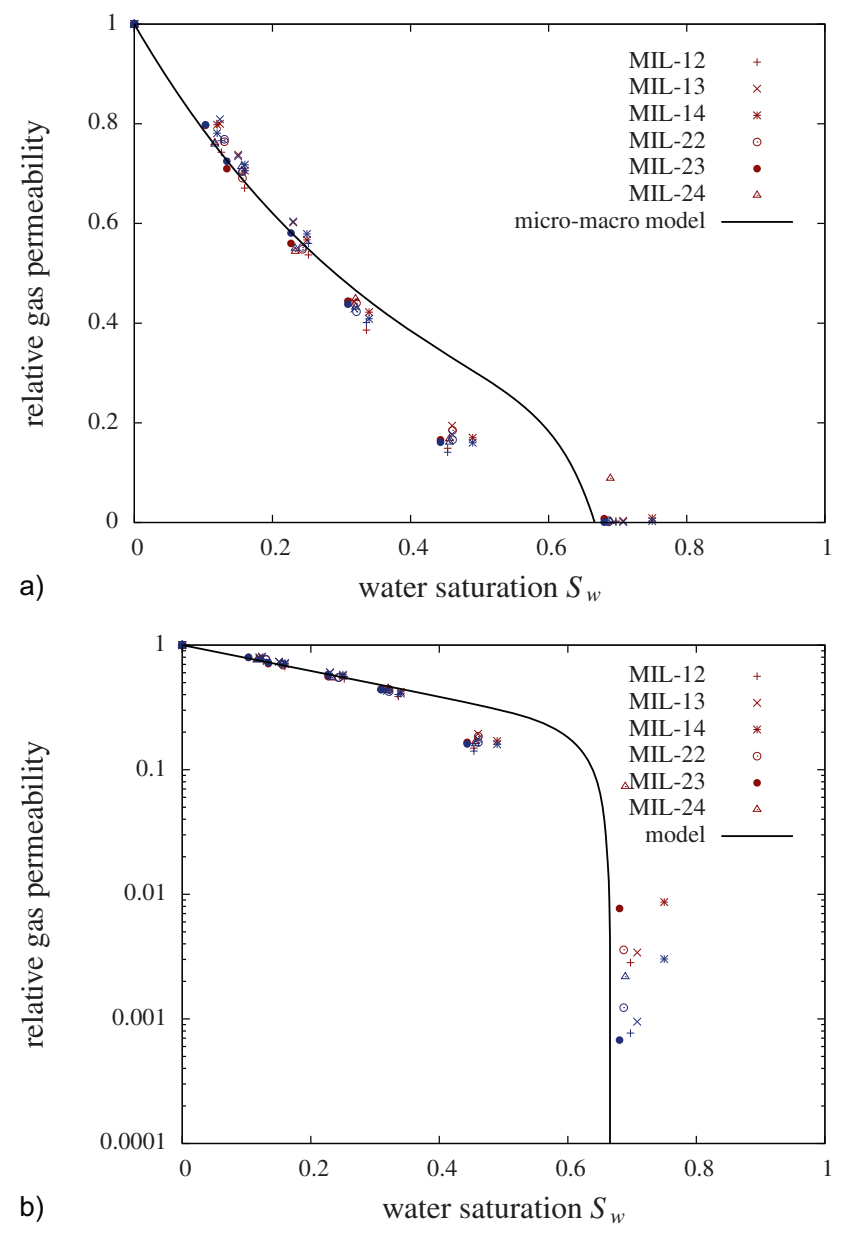

Figure 10

Comparison of the micro-macro model in Equation (30) to the measured relative gas permeabilities. a) Normal scale; b) logscale.

the equivalent grain permeability (Eq. 22), scales as $e_{\text {med }}^{2} \times e_{\text {med }} / R$ with $e_{\text {med }} \ll R$.

\subsection{Comparison to Experimental Results}

The explicit estimate Equation (30) of the homogenised gas permeability is compared to experimental results in Figure 10, under the hypothesis that the interface water saturation $s$ is equal to the total water saturation $S_{w}$ and that the high porosity region volume fraction is equal to the total porosity $\phi$. Based on the experimental results, we used $\phi=0.11$ (Tab. 2), $e_{\min }=0.6 \mathrm{~nm}$ and $e_{\max }=360 \mathrm{~nm}$ (Fig. 6) such that no parameter has to be fitted. The radius $R$ of the grains has no impact on the relative permeability curve as results are normalised by the permeability corresponding to the dry case. It is emphasised that the threshold saturation $S_{w}^{c}$ is an output of the model. In the present case, where spheres have been simply chosen to represent the grains, $S_{w}^{c}=2 / 3$.

The agreement is good for water saturations below 0.3 , but beyond that the model tends to over-estimate the relative gas permeability due to an inflexion point in the curve of the model. The percolation threshold $S_{w}^{c}=2 / 3$ given by the self-consistent scheme for spherical grains and $S_{w}=s$ is slightly below the actual critical water saturation $(\approx 0.7)$. Choosing prolate (elongated) spheroids with aspect ratio $\omega=2$ to describe the grains would have led to a better agreement for the critical water saturation. Note however that all these remarks are very sensitive to the choice of the $s-S_{w}$ relation, which here has a priori been set to $s=S_{w}$ for the sake of simplicity.

\section{CONCLUSION}

An experimental characterisation of the porosity, capillary pressure curve, dry and relative gas permeabilities has been carried out on a set of samples of a low permeability caprock. Two methods have been used to measure the porosity: with water or gas. Water porosities, around $9 \%$, are systematically below gas porosities, around $11 \%$. This difference could be attributed to trapped gas in the "water porosity" measurements or to gas sorption on pore walls when performing "gas porosity" measurements, and has been observed on other materials such as argillite. The interpretation of the capillary pressure curves as approximate pore size distributions indicates that $2 / 3$ of the pore volume is comprised of very small pores, below $100 \mathrm{~nm}$ opening. Based on SEM imaging, these small sizes are attributed to the clay content in high porosity regions, to canaliculi crossing carbonate aggregates and to joints around silts.

The Klinkenberg-corrected intrinsic gas permeabilities on dry samples are low, around $2 \times 10^{-19} \mathrm{~m}^{2}$ for most samples. The relative gas permeability measurements demonstrate a strong repeatability as all samples lie on the same curve. The low confining pressures which have been used (9 $\mathrm{MPa} \approx$ as in-situ and $2 \mathrm{MPa}$ ) did not have a significant impact on the porosity or relative gas permeability measurements. The important feature of the relative permeability curve is a gas permeability cut-off for critical water saturations around $0.71 \pm 0.04$. This has strong implications for gas storage applications, since it implies that the caprock can prevent gas flow even in the case of low desaturation. It means that a caprock exhibits two interesting features in term of confinement properties. The first is the standard one which consists of a static barrier related to the threshold capillary pressure value. The second one evidenced by this work consists of a dynamic barrier, related to the gas relative 
permeability curve shape, which first prevents and then delays the gas flow at low saturations.

The proposed micro-mechanical model relies on a strong simplification of the microstructure organisation. Based on several SEM images, gas flow has been assumed to be controlled by hydraulic interfaces corresponding to canaliculi surrounding impermeable carbonate aggregates or joints along impermeable silts. These interfaces have been assumed to connect high porosity regions which are partially clay-filled. The contribution of the hydraulic interfaces has been taken into account thanks to a composite grain model recently proposed in the literature. Based on a simplification of the Newtonian flow problem into an equivalent heterogeneous Darcy problem, the self-consistent scheme has been used to assess the macroscopic effective gas permeability. The model includes an interface aperture size distribution and allows study of the effect of progressive water saturation. The percolation threshold naturally arising in the self-consistent scheme allowed modeling of the critical water saturation corresponding to the gas permeability cut-off. A closed-form model has been proposed, whose parameters may all be deduced from porosimetry measurements. The agreement with experimental data is fair for the relative gas permeability at low water saturations $\left(S_{w}<0.3\right)$ and for the value of the critical water saturation. However, the relative gas permeability is still poorly modeled at intermediate water saturations $\left(0.3<S_{w}<0.6\right)$. Further improvements could be made based on a more accurate description of the progressive and perhaps differential localisation of the water in the interfaces or in the high porosity regions.

\section{ACKNOWLEDGMENTS}

The authors acknowledge financial support from ENGIE $E \& P$ International and Storengy. The authors would like to thank Bertrand van de Moortèle and Gilles Dromart from ENS Lyon for the petrographic analysis of the tested rock.

\section{REFERENCES}

1 Dana E., Skoczylas F. (1999) Gas relative permeability and pore structure of sandstones, International Journal of Rock Mechanics and Mining Sciences 36, 613-625.

2 Dana E., Skoczylas F. (2002) Experimental study of two-phase flow in three sandstones. I. Measuring relative permeabilities during two-phase steady-state experiments, International Journal of Multiphase Flow 28, 1719-1736.

3 Dana E., Skoczylas F. (2002) Experimental study of two-phase flow in three sandstones. II. Capillary pressure curve measurement and relative permeability pore space capillary models, International Journal of Multiphase Flow 28, 1965-1981.
4 Chen W., Liu J., Brue F., Skoczylas F., Davy C.A., Bourbon X., Talandier J. (2012) Water retention and gas relative permeability of two industrial concretes, Cement and Concrete Research 42, 1001-1013.

5 Duan Z., Davy C.A., Agostini F., Jeannin L., Troadec D., Skoczylas F. (2014) Gas recovery potential of sandstones from tight gas reservoirs, International Journal of Rock Mechanics \& Mining Sciences 65, 75-85.

6 Chen X.T., Caratini G., Davy C.A., Troadec D., Skoczylas F. (2013) Coupled transport and poro-mechanical properties of a heat-treated mortar under confinement, Cement and Concrete Research 49, 10-20.

7 Davy C.A., Skoczylas F., Barnichon J.D., Lebon P. (2007) Permeability of macro-cracked argillite under confinement: Gas and water testing, Physics and Chemistry of the Earth 32, 667-680.

8 Lion M., Skoczylas F., Ledésert B. (2004) Determination of the main hydraulic and poro-elastic properties of a limestone from Bourgogne, France, International Journal of Rock Mechanics \& Mining Sciences 41, 915-925.

9 Nadah J., Bignonnet F., Davy C.A., Skoczylas F., Troadec D., Bakowski S. (2013) Microstructure and poro-mechanical performance of Haubourdin chalk, International Journal of Rock Mechanics \& Mining Sciences 58, 149-165.

10 Klinkenberg L.J. (1941) The permeability of porous media to liquids and gases. In API Drilling and Production Practices, New York, 200-213, API 11th mid-year meeting, Tulsa.

11 Bignonnet F. (2014) Caractérisation expérimentale et modélisation micromécanique de la perméabilité et de la résistance de roches argileuses. PhD Thesis, Université Paris Est.

12 Zaoui A. (2002) Continuum micromechanics: survey, Journal of Engineering Mechanics 128, 808-816.

13 Barthélémy J.-F. (2009) Effective permeability of media with a dense network of long and micro fractures, Transp. Porous Media 76, 153-178.

14 Dormieux L., Kondo D. (2004) Approche micromécanique du couplage perméabilité-endommagement, C. R. Mécanique 332, 135-140.

15 Fokker P. (2001) General anisotropic effective medium theory for the effective permeability of heterogeneous reservoirs, Transport in Porous Media 44, 205-218.

16 Lemarchand E., Davy C.A., Dormieux L., Chen W., Skoczylas F. (2009) Micromechanics contribution to coupled transport and mechanical properties of fractured geomaterials, Transport in porous media $\mathbf{7 9}, 335-358$.

17 Lemarchand E., Davy C.A., Dormieux L., Skoczylas F. (2010) Tortuosity effects in coupled advective transport and mechanical properties of fractured geomaterials, Transport in porous media 84, 1-19.

18 Cariou S. (2010) Couplage hydro-mécanique et transfert dans l'argilite de Meuse/Haute-Marne : approches expérimentale et multiéchelle, PhD Thesis, École Nationale des Ponts et Chaussées.

19 Dormieux L., Jeannin L., Gland N. (2011) Homogenized models of stress-sensitive reservoir rocks, Int. J. Eng. Science 49, 386-396.

20 Kröner E. (1978) Self-consistent scheme and graded disorder in polycrystal elasticity, Journal of Physics F 8, 2261-2267.

21 Bernard D., Nielsen Ø., Salvo L., Cloetens P. (2005) Permeability assessment by 3D interdentritic flow simulations on microtomography mappings of Al-Cu alloys, Materials Science and Engineering 392, 112-120. 
22 Bignonnet F., Dormieux L. (2014) FFT-based bounds on the permeability of complex microstructures, International Journal for Numerical and Analytical Methods in Geomechanics 38, 1707-1723.

23 Jung Y., Torquato S. (2005) Fluid permeabilities of triply periodic minimal surfaces, Physical Review E 72, 056319.

24 Monchiet V., Bonnet G., Lauriat G. (2009) A FFT-based method to compute the permeability induced by a Stokes slip flow through a porous medium, Comptes Rendus de Mécanique 337, 192-197.

25 Nguyen T.K., Monchiet V., Bonnet G. (2013) A Fourier based numerical method for computing the dynamic permeability of periodic porous media, European Journal of Mechanics B/Fluids 37, 90-98.

26 Tardif d'Hamonville P., Ern A., Dormieux L. (2007) Finite element evaluation of diffusion and dispersion tensors in periodic porous media with advection, Computational Geosciences 11, 43-58.

27 Tölke J., Baldwin C., Mu Y., Derzhi N., Fang Q., Grader A., Dvorkin J. (2010) Computer simulations of fluid flow in sediment: from images to permeability, The Leading Edge 29, 68-74.

28 Wiegmann A. (2007) Computation of the permeability of porous materials from their microstructure by FFF-Stokes. Berichte des Fraunhofer ITWM, 129.

29 Dullien F.A.L. (1992) PorousMedia: Fluid Transport and Pore Structure. Number ISBN: 978-0-12-223651-8. Academic Press, San Diego, second edition.
30 Fredlund D.G., Xing A. (1994) Equations for the soil-water characteristic curve, Can. Geotech. J. 31, 521-532.

31 Mualem Y. (1976) A new model for predicting the hydraulic conductivity of unsaturated porous media, Water Resour. Res. 12, 513-522.

32 van Genuchten M.T. (1980) A closed-form equation for the hydraulic conductivity of unsaturated soils, Soil Sci. Soc. Am. J. 44, 892-898.

33 Ene H., Sanchez-Palencia E. (1975) Equations et phénomènes de surface pour l'écoulement dans un modèle de milieu poreux, Journal de Mécanique, 73-108.

34 He Z., Dormieux L., Lemarchand E., Kondo D. (2012) A poroelastic model for the effective behavior of granular materials with interface effect, Mechanics Research Communications 43, 41-45.

35 Boutin C. (2000) Study of permeability by periodic and selfconsistent homogenisation, European Journal of Mechanics A/Solids 19, 603-632.

36 Happel J. (1958) Viscous flow in multiparticle systems: slow motion of fluids relative to beds of spherical particles, AIChE J. 4, 197-201.
Manuscript submitted in May 2015

Manuscript accepted in May 2016

Published online in June 2016

Cite this article as: F. Bignonnet, Z. Duan, P. Egermann, L. Jeannin and F. Skoczylas (2016). Experimental Measurements and MultiScale Modeling of the Relative Gas Permeability of a Caprock, Oil Gas Sci. Technol 71, 55. 